\title{
ON THE LOGARITHMIC MEANS OF THE SUCCESSIVELY DERIVED CONJUGATE SERIES OF A FOURIER SERIES
}

\section{SULAXANA KUMARI}

1. Definition [6]. A series $\sum c_{n}$ is said to be summable $(R, \log \omega, k)$ or summable $(R, k)$, to sum $s$, if

$$
R_{k}(\omega)=\frac{1}{(\log \omega)^{k}} \sum_{n<\omega}\left(\log \frac{\omega}{n}\right)^{k} c_{n}
$$

tends to a limit $s$, as $\omega \rightarrow \infty . R_{k}(\omega)$ is called the $(R, \log \omega, k)$ mean of $\sum c_{n}$.

Let the Fourier series of a function $f(\theta) \in L$ over $(-\pi, \pi)$ and periodic outside this range with period $2 \pi$, be

(1.1) $f(\theta) \sim \frac{a_{0}}{2}+\sum_{n=1}^{\infty}\left(a_{n} \cos n \theta+b_{n} \sin n \theta\right)=\frac{A_{0}}{2}+\sum_{n=1}^{\infty} A_{n}(\theta)$.

The series conjugate to the above Fourier series is

$$
\sum_{n=1}^{\infty}\left(b_{n} \cos n \theta-a_{n} \sin n \theta\right)=\sum_{n=1}^{\infty} B_{n}(\theta)
$$

and the $r$ th derived series of the above conjugate series is

$$
\sum_{n=1}^{\infty} \frac{d^{r}}{d \theta^{r}}\left(b_{n} \cos n \theta-a_{n} \sin n \theta\right) .
$$

Suppose that $P(t)$ is a polynomial of $(r-1)$ th degree in $t$, such that

$$
h(t)=\frac{1}{2 t^{r}}\left[\{f(x+t)-P(t)\}-(-1)^{r}\{f(x-t)-P(-t)\}\right]
$$

is integrable $(L)$ over $(-\pi, \pi)$ and is defined by periodicity outside this range with period $2 \pi$.

In what follows we shall write

$$
\begin{aligned}
\psi(t) & =\frac{1}{2}\{f(x+t)-f(x-t)\} \\
\Psi_{\alpha}(t) & =\frac{1}{\Gamma(\alpha)} \int_{0}^{t}(t-u)^{\alpha-1} \psi(u) d u, \quad \alpha>0 \\
\psi_{\alpha}(t) & =\frac{\Gamma(\alpha+1)}{t^{\alpha}} \Psi_{\alpha}(t)
\end{aligned}
$$

Received by the editors January 31, 1962. 


$$
\begin{aligned}
& \bar{\Psi}_{\alpha}(t)=\frac{1}{\Gamma(\alpha)} \int_{t}^{\pi}\left(\log \frac{u}{t}\right)^{\alpha-1} \frac{\psi(u)}{u} d u, \quad \alpha>0, \\
& \Psi_{\alpha}(t)=\Gamma(\alpha+1)\left(\log \frac{1}{t}\right)^{-\alpha} \bar{\Psi}_{\alpha}(t),
\end{aligned}
$$

and define $\theta_{\alpha}(t), \bar{\Theta}_{\alpha}(t), \bar{\theta}_{\alpha}(t), \bar{H}_{\alpha}(t)$ and $\bar{h}_{\alpha}(t)$ in a similar way.

Also we shall denote by $R_{0}^{\delta}(\omega)$ and $R_{r}^{\delta}(\omega)$ the $(R, \delta)$ means of (1.2) and (1.3), respectively and by $B^{\delta}(\omega)$ the $(R, \delta)$ mean of the conjugate series of $h(t)$.

2. It is known that if $\theta_{\alpha}(t) \rightarrow 0$ as $t \rightarrow 0$, for $\alpha \geqq 0$, then

$$
C_{\alpha}(n) \sim \frac{2 l}{\pi} \log n
$$

where $C_{\alpha}(n)$ denotes the $(C, \alpha)$ mean of the conjugate series (1.2) at $\theta=x$. This result for $\alpha=0$ is the classical result of Lukàcs [2] and for $\alpha>0$ it has been obtained by Obrechkoff [5].

Considering logarithmic means instead of Cesàro means, we find that if for $p>-1, \alpha \geqq 0$,

$$
\int_{t}^{\pi} \frac{\left|\bar{\theta}_{\alpha}(u)\right|}{u} d u=o\left\{\left(\log \frac{1}{t}\right)^{p+1}\right\}, \text { as } t \rightarrow 0,
$$

then

$$
R_{0}^{\alpha}(\omega)=\frac{2 l}{\pi(\alpha+1)} \log \omega+o\left\{(\log \omega)^{p+1}\right\}, \text { as } \omega \rightarrow \infty .
$$

(See Lemma 2 of the present paper.) For $p=0$ this gives a result analogous to (2.1), which, although not explicitly mentioned, may also be obtained from a result of Misra [3].

For the first derived series of the conjugate series, Mohanty and Nanda [4] have recently proved the following theorem:

TheOREM A. If

$$
\begin{aligned}
\int_{t}^{\pi}\left|\{f(x+u)+f(x-u)-2 f(x)\} /\left(4 \sin \frac{u}{2}\right)-l\right| u^{-1} d u & \\
& =o\left(\log \frac{1}{t}\right), \text { as } t \rightarrow 0
\end{aligned}
$$

then $\lim _{n \rightarrow \infty}\left(\sigma_{2 n}-\sigma_{n}\right)=l / \pi \log 2$, where $\sigma_{n}$ denotes the $(R, \log n, 1)$ mean of the first derived conjugate series, at $\theta=x$. 
The object of the present paper is to generalize this theorem to the case of the $r$ th derived series of the conjugate series and to obtain a more refined result by replacing $\sigma_{2 n}$ and $\sigma_{n}$ by $R_{r}^{r}(\lambda \omega)$ and $R_{r}^{r}(\omega)$ respectively and also by replacing the condition of the theorem by a set of weaker conditions. Thus we prove the following theorem which gives a more far-reaching result.

THEOREM 1. If

$$
\int_{t}^{\pi}|h(u)-l| u^{-1} d u=o\left\{\left(\log \frac{1}{t}\right)^{r}\right\}, \text { as } t \rightarrow 0, \text { for } r \geqq 1,
$$

and

$$
\int_{t}^{\pi}\left|\bar{h}_{r-1}(u)-s\right| u^{-1} d u=o\left(\log \frac{1}{t}\right), \text { as } t \rightarrow 0, \text { for } r \geqq 1,
$$

then

$$
\left[R_{r}^{r}(\lambda \omega)-R_{r}^{r}(\omega)\right] \sim \frac{2 l}{\pi} \frac{r !}{r+1} \log \lambda, \quad \lambda>1 .
$$

I am indebted to Professor B. N. Prasad for his kind encouragement during the preparation of this paper.

3. We require the following lemmas for the proof of our theorem:

LEMMA $1[8]$. If

$$
M_{p}(t)=\int_{0}^{1}\left(\log \frac{1}{u}\right)^{p} \sin u t d u, \quad p>-1,
$$

then

$$
\frac{d}{d t}\left\{t M_{p}(t)\right\}=p M_{p-1}(t), \quad p>0 .
$$

LEMMA 2. If for $p>-1, \alpha \geqq 0$,

$$
\int_{t}^{\pi} \frac{\left|\bar{\theta}_{\alpha}(u)\right|}{u} d u=o\left\{\left(\log \frac{1}{t}\right)^{p+1}\right\} \text {, as } t \rightarrow 0
$$

then

$$
R_{0}^{\alpha}(\omega)=\frac{2 l}{\pi(\alpha+1)} \log \omega+o\left\{(\log \omega)^{p+1}\right\}, \text { as } \omega \rightarrow \infty .
$$

Proof of Lemma 2. Suppose that $h=[\alpha]$. We have by well-known arguments 


$$
\begin{aligned}
R(\omega)= & \sum_{n<\omega} B_{n}(x)=\frac{1}{\pi} \int_{0}^{\pi} \psi(t) \cot \frac{t}{2}(1-\cos \omega t) d t+o(1) \\
= & \frac{1}{\pi} \int_{0}^{\pi} \theta(t) \cot \frac{t}{2}(1-\cos \omega t) d t \\
& +\frac{l}{\pi} \int_{0}^{\pi} \cot \frac{t}{2}(1-\cos \omega t) d t+o(1) \\
= & \frac{1}{\pi} \int_{0}^{\pi} \theta(t)\left(\cot \frac{t}{2}-\frac{2}{t}\right)(1-\cos \omega t) d t \\
& +\frac{2}{\pi} \int_{0}^{\pi} \theta(t) \frac{1-\cos \omega t}{t} d t+\frac{2 l}{\pi} \log \omega+O(1) \\
= & \frac{2}{\pi} \int_{0}^{\pi} \theta(t) \frac{1-\cos \omega t}{t} d t+\frac{2 l}{\pi} \log \omega+O(1) . \\
= & \frac{2 \omega}{\pi} \int_{0}^{\pi} \bar{\Theta}_{1}(t) \sin \omega t d t+\frac{2 l}{\pi} \log \omega+O(1) .
\end{aligned}
$$

Considering first the case when $\alpha$ is nonintegral, we have

$$
\begin{aligned}
R_{0}^{\alpha}(\omega)= & \frac{1}{(\log \omega)^{\alpha}} \sum_{n<\omega}\left(\log \frac{\omega}{n}\right)^{\alpha} B_{n}=\frac{\alpha}{(\log \omega)^{\alpha}} \int_{1}^{\omega}\left(\log \frac{\omega}{x}\right)^{\alpha-1} \frac{R(x)}{x} d x \\
= & \frac{2 l}{\pi} \frac{\alpha}{(\log \omega)^{\alpha}} \int_{1}^{\omega}\left(\log \frac{\omega}{x}\right)^{\alpha-1} \frac{\log x}{x} d x \\
& +\frac{2 \alpha}{\pi} \frac{1}{(\log \omega)^{\alpha}} \int_{1}^{\omega}\left(\log \frac{\omega}{x}\right)^{\alpha-1} d x \int_{0}^{\pi} \bar{\Theta}_{1}(t) \sin x t d t+O(1) \\
= & \frac{2 l}{\pi} \frac{\log \omega}{\alpha+1}+\frac{2 \alpha}{\pi(\log \omega)^{\alpha}} \int_{0}^{\pi} \bar{\Theta}_{1}(t) d t \int_{1}^{\omega}\left(\log \frac{\omega}{x}\right)^{\alpha-1} \sin x t d t \\
& +O(1) \\
= & \frac{2 l}{\pi} \frac{\log \omega}{\alpha+1}+\frac{2 \alpha \omega}{\pi(\log \omega)^{\alpha}} \int_{0}^{\pi} \overline{\Theta_{1}(t) M_{\alpha-1}(\omega t) d t} \\
& -\frac{2 \alpha \omega}{\pi(\log \omega)^{\alpha}} \int_{0}^{\pi} \overline{\Theta_{1}}(t) \int_{0}^{1 / \omega}\left(\log \frac{1}{v}\right)^{\alpha-1} \sin (\omega t v) d v+O(1) \\
= & \frac{2 l}{\pi} \frac{\log \omega}{\alpha+1}+\frac{2 \alpha \omega}{\pi(\log \omega)^{\alpha}} \int_{0}^{\pi} \frac{\bar{\Theta}_{1}(t)}{t} t M_{\alpha-1}(\omega t) d t+O(1)
\end{aligned}
$$

where 


$$
M_{p}(t)=\int_{0}^{1}\left(\log \frac{1}{u}\right)^{p} \sin u t d u, \quad p>-1
$$

Thus, integrating by parts,

$$
\begin{aligned}
R_{0}^{\alpha}(\omega)= & \frac{2 l}{\pi} \frac{\log \omega}{\alpha+1}+c \frac{\omega}{(\log \omega)^{\alpha}} \int_{0}^{\pi} \bar{\Theta}_{h+1}(t) M_{\alpha-h-1}(\omega t) d t+O(1) \\
= & \frac{2 l}{\pi} \frac{\log \omega}{\alpha+1}+c_{1} \frac{\omega}{(\log \omega)^{\alpha}} \int_{0}^{\pi} M_{\alpha-h-1}(\omega t) \\
\cdot & \left\{\int_{t}^{\pi}\left(\log \frac{u}{t}\right)^{h-\alpha} \frac{\bar{\Theta}_{\alpha}(u)}{u} d u\right\} d t+O(1) \\
& =\frac{2 l}{\pi} \frac{\log \omega}{\alpha+1}+\frac{c_{1} \omega}{(\log \omega)^{\alpha}} \int_{0}^{\pi} \frac{\vec{\Theta}_{\alpha}(u)}{u} \\
1) & \left.=\frac{2 l}{\pi} \frac{\log \omega}{\alpha+1}+\frac{c_{1}}{(\log \omega)^{\alpha}}\left(\log \frac{u}{t}\right)^{h-\alpha} M_{\alpha-h-1}(\omega t) d t\right\} d u+O(1) \\
& \left.\cdot\left\{\int_{0}^{1 / \omega}+\int_{1 / \omega}^{\pi}\right\} \frac{\vec{\Theta}_{\alpha}(u)}{u}(1-\cos \omega u) d u\right]+O(1) \\
= & \frac{2 l}{\pi} \frac{\log \omega}{\alpha+1}+\frac{c_{1}}{(\log \omega)^{\alpha}}\left[J_{1}-J_{2}\right]+O(1), \text { say. }
\end{aligned}
$$

Now

$$
\begin{aligned}
J_{1}= & o\left[\int_{0}^{1 / \omega} \frac{\left|\bar{\Theta}_{\alpha}(u)\right|}{u} \omega^{2} u^{2} d u\right] \\
= & o\left[\omega \int_{0}^{1 / \omega} \frac{\left|\bar{\Theta}_{\alpha}(u)\right|}{u} \cdot u d u\right] \\
= & O\left[\omega\left\{-u \int_{u}^{\pi} \frac{\left|\bar{\Theta}_{\alpha}(u)\right|}{u} d u\right\}_{u=0}^{u=1 / \omega}\right. \\
& \left.\quad+\omega\left\{\int_{0}^{1 / \omega} \int_{u}^{\pi} \frac{\left|\bar{\Theta}_{\alpha}(u)\right|}{u} d u\right\}\right] \\
= & o\left[(\log \omega)^{\alpha+p+1}\right] .
\end{aligned}
$$

Again 


$$
J_{2}=O\left[\int_{1 / \omega}^{\pi} \frac{\left|\vec{\Theta}_{\alpha}(u)\right|}{u} d u\right]=o\left\{(\log \omega)^{\alpha+p+1}\right\}
$$

and the result follows from (3.1), (3.2) and (3.3).

For the case when $\alpha$ is an integer, the proof becomes simpler.

LEMMA 3 [3]. If

$$
\int_{i}^{\pi}\left|\bar{\Theta}_{\alpha}(u)\right| u^{-1} d u=o\left\{\left(\log \frac{1}{t}\right)^{\alpha+1}\right\} \text {, as } t \rightarrow 0, \text { for } \alpha \geqq 0,
$$

then

$$
\lim _{\omega \rightarrow \infty}\left[R_{0}^{\alpha+1}(\lambda \omega)-R_{0}^{\alpha+1}(\omega)\right]=\frac{2 l}{\pi(\alpha+2)} \log \lambda, \quad \lambda>1 .
$$

Lemma 4 [1].

$$
R_{r}^{r}(\omega)=(-1)^{r}\left[\alpha_{0} B^{r}(\omega)+\alpha_{1} \frac{B^{r-1}(\omega)}{\log \omega}+\cdots+\alpha_{r} \frac{B^{0}(\omega)}{(\log \omega)^{r}}\right],
$$

where $\alpha_{0}=(-1)^{r} r$ ! and $\alpha_{p}$ 's for $p=1,2, \cdots, r$, are constants independent of $\omega$.

Lemma 5 [7]. Let $V(\omega)$ and $W(\omega)$ be two positive nondecreasing functions of $\omega$. Let

$$
U_{r}(\omega)=\{V(\omega)\}^{1-p / k}\{W(\omega)\}^{p / k} .
$$

Then (i) $h^{k}(\omega)=o\{W(\omega)\}$ and (ii) $h(\omega)=o\{V(\omega)\}$ together imply that $h^{p}(\omega)=o\left\{U_{p}(\omega)\right\}$ for $o<p<k$, where $h^{p}(\omega)$ denotes the $(R, p)$ mean of $h(\omega)$. Also the result remains true if in any one of the conditions (i) and (ii), " $O$ " is replaced by " $O$ ".

4. Proof of Theorem 1. By Lemma 4 we have

$$
R_{r}^{r}(\omega)=r ! B^{r}(\omega)+(-1)^{r}\left[\alpha_{1} \frac{B^{r-1}(\omega)}{\log \omega}+\cdots+\alpha_{r} \frac{B^{0}(\omega)}{(\log \omega)^{r}}\right] .
$$

Also by Lemma 2 and by the first condition of the theorem

$$
B^{0}(\omega)=o\left\{(\log \omega)^{r}\right\}, \quad \text { for } r>1,
$$

and by the second condition

$$
\begin{aligned}
B^{r-1}(\omega) & =\frac{2 l}{\pi r} \log \omega+o(\log \omega) \\
& =O(\log \omega) .
\end{aligned}
$$


Now by Lemma 5 we have

$$
\begin{gathered}
B^{1}(\omega)=o\left\{(\log \omega)^{r-1}\right\}, \\
B^{2}(\omega)=o\left\{(\log \omega)^{r-2}\right\}, \\
\cdot \cdot \cdot \cdot \cdot \cdot \cdot \cdot, \\
B^{r-2}(\omega)=o\left\{(\log \omega)^{2}\right\} .
\end{gathered}
$$

Thus, supposing that $r>1$,

$$
\begin{aligned}
\begin{array}{r}
R_{r}^{r}(\lambda \omega)-R_{r}^{r}(\omega) \\
=
\end{array} & r !\left\{B^{r}(\lambda \omega)-B^{r}(\omega)\right\}+(-1)^{r} \alpha_{1}\left\{\frac{B^{r-1}(\lambda \omega)}{\log (\lambda \omega)}-\frac{B^{r-1}(\omega)}{\log \omega}\right\} \\
& +\cdots+(-1)^{r} \alpha_{r}\left\{\frac{B^{0}(\lambda \omega)}{\left\{\log (\lambda \omega)^{r}\right.}-\frac{B^{0}(\omega)}{(\log \omega)^{r}}\right\} \\
= & r !\left\{B^{r}(\lambda \omega)-B^{r}(\omega)\right\}+(-1)^{r} \frac{\alpha_{1} 2 l}{\pi r}\left\{\frac{\log (\lambda \omega)}{\log (\lambda \omega)}-\frac{\log \omega}{\log \omega}\right\} \\
& +o\left\{\frac{\log (\lambda \omega)}{\log (\lambda \omega)}\right\}+o\left\{\frac{\log \omega}{\log \omega}\right\}+\cdots+o\left\{\frac{\{\log (\lambda \omega)\} r}{\{\log (\lambda \omega)\}^{r}}\right\}+o\left\{\frac{(\log \omega)^{r}}{(\log \omega)^{r}}\right\} \\
= & r !\left\{B^{r}(\lambda \omega)-B^{r}(\omega)\right\}+o(1)=r ! \frac{2 l}{\pi} \frac{\log \lambda}{r+1}+o(1)
\end{aligned}
$$

by Lemma 3. This completes the proof.

\section{REFERENCES}

1. S. Kumari, On the logarithmic summability of the successively derived series of a Fourier series and of its conjugate series, Indian J. Math. 1 (1959), 87-101.

2. F. Lukàcs, Über die Bestimmung des Sprunges einer Funktion aus ihrer Fourierreihe, J. Reine Angew. Math. 150 (1920), 107-112.

3. M. L. Misra, On the determination of the jump of a function by its Fourier coefficients, Quart. J. Math. Oxford Ser. (2), 18 (1947), 147-156.

4. R. Mohanty and M. Nanda, On the logarithmic mean of the derived conjugate series of a Fourier series, Proc. Amer. Math. Soc. 7 (1956), 397-400.

5. N. Obrechkoff, Sur la sommation des séries trigonometriques de Fourier par les moyennes arithmetiques, Bull. Soc. Math. France 62 (1934), 84-109, (Suit et fin) 167-184.

6. M. Riesz, Sur les séries de Dirichlet et les séries entières, C. R. Acad. Sci. Paris 149 (1908), 309-312.

7. - Sur un théorème de la moyenne et ses applications, Acta Sci. Math. Szeged) 1 (1923), 114-126.

8. F. T. Wang, On the summability of conjugate Fourier series by Riesz's logarithmic means, Tôhoku Math. J. 40 (1935), 393-397.

UNIVERSITY OF GORAKHPUR, GORAKHPUR, INDIA 